\section{BIBLIOGRAFÍA}

AMARAL FRANCO, J. -1986- Juniperus. In Castroviejo, S., M. Laínz, G. López González, P. Montserrat, F. Muñoz Garmendia, L. Paiva y L. Villar (eds.). Flora Iberica, Vol. I Real Jard. Bot., Madrid, C.S.I.C

FERNANDEZ GALIANO, E. -1987- Juniperus. In Valdés, B., S. Talavera y E. Fernández Galiano (eds.). Flora Vascular de Andalucía Occidental. Ketres. Barcelona.

LEBRETON, Ph. -1983- Nouvelles données sur la distribution au Portugal et en Espagne des sousespèces du genévrier de Phénicie (Juniperus phoenicea L.). Agronomia Lusitanica, 42: 5662.
LEBRETON, Ph \& S. THIVEND -1981- Sur une sous-espèce du Genévrier de Phénicie Juniperus phoenicea L., définie à partir de critères biochimiques. Naturalia Monspeliensia, 47: 112.

LEBRETON, Ph \& RIVERA, D. -1988- Analyse du taxon Juniperus phoenicea L. sur des bases biochimiques et biométriques. Naturalia Monspeliensia, 53: 17-41.

Aceptado para su publicación en Junio de 1995

Dirección de los autores. Departamento de Biología Vegetal y Ecología. Universidad de Sevilla. Apdo. 1095. 41080 Sevilla.

\title{
36. APORTACIONES A LA FLORA DE ANDALUCÍA: SIERRA MORENA (CÓRDOBA)
}

Manuel MELENDO LUQUE, Eusebio CANO CARMONA y Francisco VALLE TENDERO

Palabras clave. Flora, corología, fitosociología, Córdoba, Andalucía.

Key words. Flora, corology, fitosociology, Córdoba, Andalusian.

Como resultado de un estudio llevado a cabo en el noreste de la provincia de Córdoba, aportamos datos florísticos que revisten diverso interés. Algunas de las citas constituyen una novedad para la provincia, en tanto que otras lo son para Andalucía occidental o Andalucía.

Amaranthus powellii S. Watson

CÓRDOBA: Cardeña, El Robledillo, 30SUH8637, $720 \mathrm{~m}$, 24-6-93, M. Melendo, GDAC 39348. Nitrófila, en cultivos de huerta. No conocemos ninguna cita anterior de esta especie para la provincia de Córdoba.

Myosotis sicula Guss.

CÓRDOBA: Venta del Charco, Alto de
Españares, 30SUH8827, 725 m, 30-4-92, M. Melendo, GDAC 39367. Borde de charcas poco profundas que no se desecan completamente durante el verano (Glycerio-Sparganion). Es la segunda cita para la provincia de Córdoba, la primera se debe a Devesa y Cabezudo (1978).

\section{Callitriche lusitanica Schotsman}

CÓRDOBA: Montoro, Arroyo Arenosillo, 30SUH7921, 280 m, 16-6-93, M. Melendo, GDAC 39369. Sumergida en arroyos de aguas someras que se desecan en verano; (comunidades de Callitricho-Batrachion). Este taxón ha sido citado con cierta frecuencia en el centro-occidente peninsular, pero existen muy pocas referencias para Andalucía (Jaén: 
Andújar, Cádiz: prox. de Tarifa y Huelva: Almonte). Primera cita para la provincia de Córdoba.

\section{Solenopsis laurentia (L.) C. Presl}

CÓRDOBA: Montoro, Arroyo Arenosillo, 30SUH7920, $280 \mathrm{~m}$, 16-6-93, M. Melendo, GDAC 39362. Se desarrolla en pequeñas cubetas y taludes húmedo-rezumantes de las orillas de arroyos poco soleados, formando parte de las comunidades de Cicendion. Primera cita para la provincia de Córdoba.

\section{Paronychia rouyana Coincy}

CÓRDOBA: Aldea Cerezo, Colchón, 30SUH9034, 680 m, 19-6-93, M. Melendo y E. Cano, GDAC 39359. Pastizales efímeros sobre suelos arenosos, silíceos y secos. Endemismo del C y E de la Península Ibérica, citado en Andalucía únicamente para Jaén. La cita que aportamos constituye la primera para Córdoba y Andalucía occidental.

Taraxacum erythrospermum Andrz. ex Besser

CÓRDOBA: Azuel, Prox. Río Yeguas, 30SUH8744, $500 \mathrm{~m}, 3-4-94$, M. Melendo, GDAC 39345. En herbazales sobre suelos frescos y mantillosos, bajo encinares ombrófilos incluibles en el Pyro-Quercetum rotundifoliae quercetosum fagineae. No ha sido citada para los territorios cordobeses de Sierra Morena, donde es muy escasa.

Coronopus squamatus (Forssk.) Asch.

CÓRDOBA: Cardeña, 30SUH8435, 760 m, 24-6-93, M. Melendo, GDAC 39351. Sobre suelos nitrificados y compactados, en comunidades de Polygono-Poetea annuae. No había sido citada para las comarcas cordobesas de Sierra Morena.

Carex flacca Schreber subsp. serrulata (Biv.) Greuter
CÓRDOBA: Cardeña, Arroyo Corcovada, 30SUH8530, $730 \mathrm{~m}$, 16-5-93, M. Melendo, GDAC 39353. Herbazales nitrófilos sobre suelos húmedos (Molinio-Arrhenatheretea). No se conocía en los territorios cordobeses de Sierra Morena.

\section{Scirpus setaceus L.}

CÓRDOBA: Azuel, Río Yeguas, 30SUH8546, 580 m, 13-6-92, M. Melendo, GDAC 39360. Comunidades sobre suelos encharcados en primavera (Cicendion). Sólo ha sido citada para Córdoba en la Campiña Alta (García Montoya y Muñoz, 1990), por lo que aportamos la segunda cita provincial y la primera para la zona cordobesa de Sierra Morena.

\section{Elatine alsinastrum L.}

CÓRDOBA: Cardeña, Arroyo Corcovada, 30SUH8530, 725 m, 21-6-93, M. Melendo, GDAC 39352. Charcas someras de aguas mesótrofas, en comunidades de Potametum trichoidis (Parvopotamion). Es la primera cita para la provincia de Córdoba. En Andalucía occidental sólo se conoce del litoral onubense.

Exaculum pusillum (Lam.) Caruel in Parl.

CÓRDOBA: Montoro, Arroyo Arenosillo, 30SUH7921, 280 m, 26-7-92, M. Melendo, GDAC 39355. Pequeñas cubetas temporalmente inundadas, en el borde del arroyo (Cicendion). Es la segunda cita para la provincia de Córdoba, la primera corresponde a la comarca del Zújar (Jiménez y Ruíz de Clavijo, 1990).

\section{Anthoxanthum ovatum Lag.}

CÓRDOBA: Montoro, Los Rasos, 30SUH8819, 400 m, 16-4-92, M. Melendo, GDAC 39365. En pastizales terofíticos sobre suelos delgados y ligeramente nitrificados. No conocemos ninguna cita anterior de esta especie para la provincia de Córdoba. 
Milium vernale $B i e b$.

CóRDoBA: Azuel, Arroyo de los Términos, 30SUH8447, 560 m, 29-4-93, M. Melendo, GDAC 39368. Herborizada en la terraza arenosa del arroyo. Es la primera cita para la provincia de Córdoba y para Andalucía occidental.

Juncus acutiflorus Ehrh. ex Hoffm. subsp. acutiflorus

CÓRDOBA: Cardeña, 30SUH8534, 730 m, 11-6-93, M. Melendo, GDAC 39358. Juncales higrófilos, incluibles en la asociación Hyperico-Juncetum acutiflori (Juncion acutiflori). Es un taxón frecuente en el territorio estudiado, aunque no conocemos ninguna cita anterior para la provincia de Córdoba.

Juncus fontanesii J. Gay in Laharpe

CÓRDOBA: Cardeña, Arroyo Corcovada, 30SUH8430, 720 m, 21-6-93, M. Melendo, GDAC 39354. Juncales sobre suelos de humedad constante (Juncion acutiflori). No conocemos ninguna cita anterior de esta especie para los territorios de Sierra Morena en Andalucía occidental.

\section{Sideritis lacaite Font Quer}

CÓRDOBA: Cardeña, Loma de Enmedio, 30SUH8530, 740 m, 8-5-93, M. Melendo, GDAC 39357. Sobre suelos graníticos delgados y secos. Caméfito sufruticoso endémico del subsector Marianense, muy poco citado.

Ononis pendula Desf. subsp. boissieri (Sirj.) Devesa

CÓRDOBA: Azuel, Prox. Arroyo de los Términos, 30SUH8447, $560 \mathrm{~m}, 13-6-92, \mathrm{M}$. Melendo, GDAC 39364. Pastizales terofíticos sobre suelos secos. No había sido citada para las comarcas cordobesas de Sierra Morena.

Ludwigia palustris (L.) Elliot

CÓRDOBA: Cardeña, Presa de Cardeña, 30SUH8736, 700 m, 1-8-93, M. Melendo,
GDAC 39350. En suelos areno-limosos temporalmente inundados (Nanocyperion flavescentis). Es la segund a cita para la comarca de los Pedroches (Jiménez y Ruíz de Clavijo, 1990).

Neotinea maculata (Desf.) Stearn

CÓRDOBA: Cardeña, Valdecañas, 30SUH9227, $600 \mathrm{~m}$, 8-4-94, M. Melendo, GDAC 39346. En alcornocales del Sanguisorbo agrimonioidis-Quercetum suberis. Es la segunda cita para la zona cordobesa de Sierra Morena (Jiménez y Ruíz de Clavijo, 1990).

Armeria genesiana Nieto Feliner subsp. genesiana

CóRDoBA: Cardeña, Prox. Arroyo Corcovada, 30SUH8530, 730 m, 16-5-93, M. Melendo, GDAC 39347. En suelos silíceos y arenosos poco desarrollados. Endemismo de los Montes de Toledo y estribaciones de Sierra Morena, conocida hasta ahora con seguridad de las provincias de Badajoz, Ciudad Real y Toledo, mientras que su existencia en la provincia de Córdoba era incierta. Confirmamos su presencia en la provincia de Córdoba y, por tanto, en Andalucía.

Ranunculus bulbosus L. subsp. aleae (Willk.) Rouy \& Fouc. var. aleae (Willk.) Burnat

CÓRDOBA: Cardeña, Prox. Arroyo Corcovada, 30SUH8430, 725 m, 16-5-93, M. Melendo, GDAC 39361. Juncales y praderas húmedas (Molinio-Arrhenatheretea). Es la primera vez que se cita esta variedad en la provincia de Córdoba.

Ranunculus ficaria L. subsp. bulbilifer Lambinon

CÓRDOBA: Venta del Charco, Arroyo Martín Gonzalo, 30SUH8528, 640 m, 12-5-92, M. Melendo, GDAC 39363. Suelos muy húmedos cercanos a los arroyos, generalmente bajo fresnedas de la asociación Ficario ranunculoidis-Fraxinetum angustifoliae. Es la 
primera cita para la provincia de Córdoba y para Andalucía de esta subespecie, cuya distribución no es bien conocida.

Verbascum giganteum Willk. subsp. martinezii Valdés

CÓRDOBA: Cardeña, Prox. presa de Cardeña, 30SUH8836, 700 m, 26-6-93, M. Melendo, GDAC 39366. Nitrófila sobre suelos silíceos y arenosos alterados, provenientes de rocas graníticas. Subespecie considerada endémica de las arenas litorales de Vejer de la Frontera y Los Caños de Meca, en el subsector Gaditano litoral del sector Gaditano-Onubense (Rivas-Martínez et al., 1991). La nuestra es la primera cita provincial de esta subespecie, para la cual se amplía así su área de distribución al subsector Marianense.

\section{Typha latifolia L.}

CÓRDOBA: Cardeña, Presa de Cardeña, 30SUH8736, 700 m, 1-8-93, M. Melendo, GDAC 39349. Espadañares en el margen del embalse (Phragmition australis). Es la primera cita para la provincia de Córdoba.

Elaeoselinum asclepium (L.) Bertol. subsp. millefolium (Boiss.) García Martín y Silvestre

CÓRDOBA: Cardeña, Barranco del Endrinar, 30SUH8346, $580 \mathrm{~m}$, 17-7-94, M. Melendo, GDAC 39356. Comunidades saxícolas en grietas de pizarras y granitos del Rumici-Dianthion lusitani. Taxon considerado hasta ahora endémico del subsector Bermejense en el sector Rondeño de la provincia Bética y del sector Aljíbico de la provincia GaditanoOnubo-Algarviense, en donde se comporta como saxícola sobre areniscas, dolomías y peridotitas (Rivas-Martínez et al., 1991). Con la cita que aportamos ampliamos su areal al subsector Marianense del sector MariánicoMonchiquense en la provincia LusoExtremadurense.

\section{BIBLIOGRAFÍA}

DEVESA, J.A. y B. CABEZUDO -1978Contribución al estudio florístico del Batolito de los Pedroches (Córdoba). Lagascalia, 8(1): 53-103.

GARCÍA MONTOYA, F. y J.M. MUÑOZ -1990Novedades corológicas para la flora de Andalucía occidental. Lagascalia, 16(1): 146168.

JIMÉNEZ, M. y E. RUÍZ DE CLAVIJO -1990Nuevas áreas para la flora de Andalucía occidental. Lagascalia, 16(1): 132-145.

RIVAS MARTÍNEZ, S., A. ASENSI, J. MOLERO y F. VALLE -1991- Endemismos vasculares de Andalucía (España). Rivasgodaya, 6: 5-76.

Aceptado para su publicación en Mayo de 1995

Dirección de los autores.- M. Melendo Luque y E. Cano Carmona: Departamento de Biología Animal, Vegetal y Ecología. Universidad de Jaén. 23071 Jaén. F. Valle Tendero: Departamento de Biología Vegetal. Facultad de Ciencias. Universidad de Granada. 18001 Granada. 